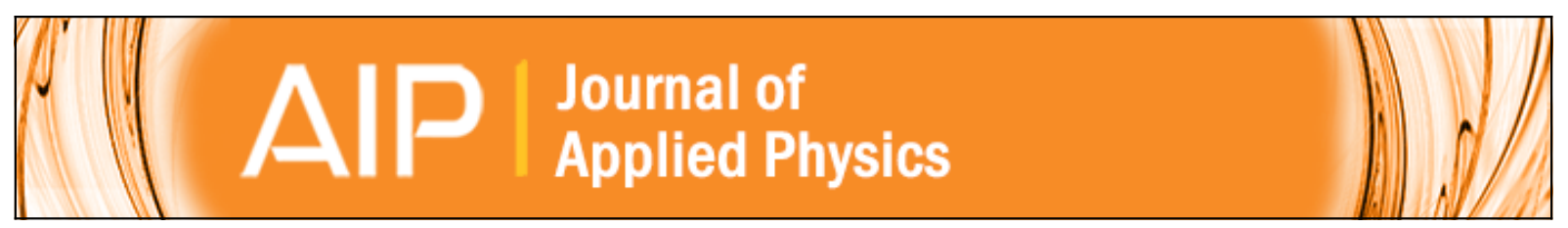

\title{
Lorentz force particle analyzer
}

Xiaodong Wang, André Thess, René Moreau, Yanqing Tan, Shangjun Dai, Zhen Tao, Wenzhi Yang, and Bo Wang

Citation: Journal of Applied Physics 120, 014903 (2016); doi: 10.1063/1.4956842

View online: http://dx.doi.org/10.1063/1.4956842

View Table of Contents: http://scitation.aip.org/content/aip/journal/jap/120/1?ver=pdfcov

Published by the AIP Publishing

\section{Articles you may be interested in}

Rapid calculation of hydrodynamic and transport properties in concentrated solutions of colloidal particles and macromolecules

Phys. Fluids 28, 011902 (2016); 10.1063/1.4939581

Direct numerical simulations of agglomeration of circular colloidal particles in two-dimensional shear flow

Phys. Fluids 28, 013304 (2016); 10.1063/1.4939501

Propagation and deposition of non-circular finite release particle-laden currents

Phys. Fluids 27, 086604 (2015); 10.1063/1.4929397

Characterization of fluids via measurement of the rotational dynamics of suspended magnetic microdiscs J. Appl. Phys. 117, 17B320 (2015); 10.1063/1.4918784

Direct Lorentz force compensation flowmeter for electrolytes

Appl. Phys. Lett. 105, 223510 (2014); 10.1063/1.4903235

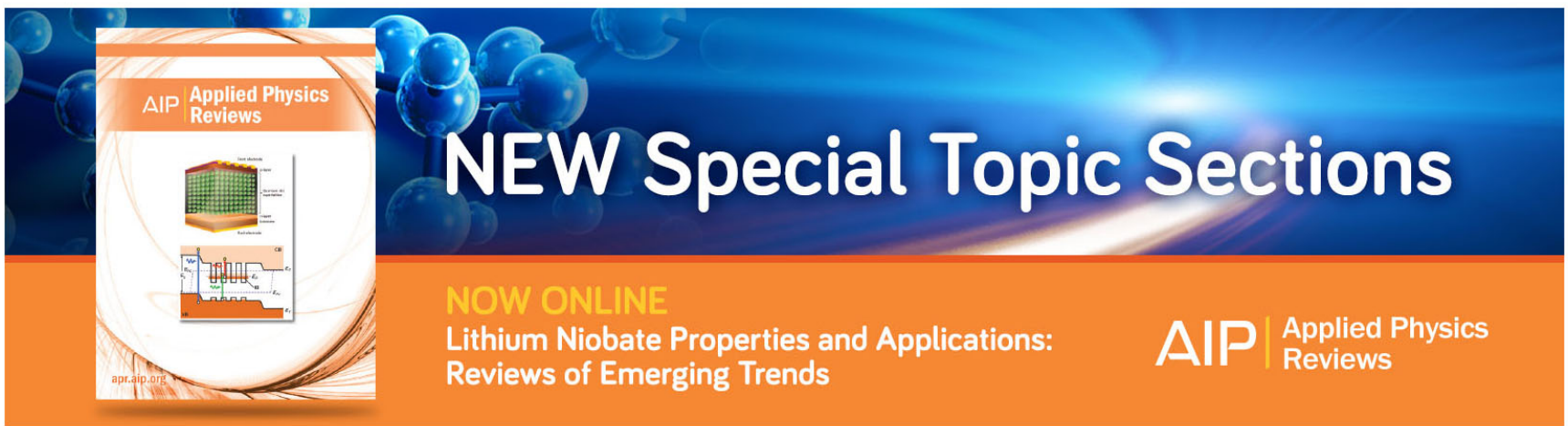




\title{
Lorentz force particle analyzer
}

\author{
Xiaodong Wang, ${ }^{1}$ André Thess, ${ }^{2}$ René Moreau, ${ }^{3}$ Yanqing Tan, ${ }^{1}$ Shangjun Dai, ${ }^{1}$ Zhen Tao, ${ }^{1}$ \\ Wenzhi Yang, ${ }^{1}$ and Bo Wang ${ }^{1}$ \\ ${ }^{1}$ University of Chinese Academy of Sciences, Yanqihu Campus, Beijing, China \\ ${ }^{2}$ DLR, Institute of Engineering Thermodynamics, Pfaffenwaldring 38-40, Stuttgart, Germany \\ ${ }^{3}$ SIMAP/EPM, Université Grenoble Alpes, St Martin d'Hères, France
}

(Received 8 November 2015; accepted 17 June 2016; published online 7 July 2016)

\begin{abstract}
A new contactless technique is presented for the detection of micron-sized insulating particles in the flow of an electrically conducting fluid. A transverse magnetic field brakes this flow and tends to become entrained in the flow direction by a Lorentz force, whose reaction force on the magnetic-field-generating system can be measured. The presence of insulating particles suspended in the fluid produce changes in this Lorentz force, generating pulses in it; these pulses enable the particles to be counted and sized. A two-dimensional numerical model that employs a moving mesh method demonstrates the measurement principle when such a particle is present. Two prototypes and a three-dimensional numerical model are used to demonstrate the feasibility of a Lorentz force particle analyzer (LFPA). The findings of this study conclude that such an LFPA, which offers contactless and on-line quantitative measurements, can be applied to an extensive range of applications. These applications include measurements of the cleanliness of high-temperature and aggressive molten metal, such as aluminum and steel alloys, and the clean manufacturing of semiconductors. Published by AIP Publishing. [http://dx.doi.org/10.1063/1.4956842]
\end{abstract}

\section{INTRODUCTION}

Let us recall three techniques that enlighten our present method of the Lorentz force particle analyzer (LFPA). The first technique is the Coulter Principle (CP). Based on developments in electric techniques for the quantification of microscopic cells, ${ }^{1}$ Coulter investigated suspensions of particles in an electrolyte in the 1940 s by forcing particles to flow through a small aperture located in an insulating tube. Two electrodes on each side of the aperture were connected to an external source of electric current to form an electrical sensing zone (ESZ). When a particle passed through the aperture, whose diameter was slightly larger than the diameters of the particles, a voltage pulse signal could be measured. This signal appeared to be proportional to the particle size. The concept behind this apparatus is known as the $\mathrm{CP} .{ }^{1-3}$

Thirty years after the $\mathrm{CP}^{1-3}$ was discovered, researchers at McGill University made a breakthrough in the analysis of molten metal quality by applying the ESZ approach to such an aggressive fluid. By applying this technique via what is now known as a liquid metal cleanliness analyzer (LiMCA), ${ }^{4}$ the number of pulses can be related to the passage of insulating particles, although the amplitude of the measured voltage is millions of times smaller than that delivered by saline aqueous solutions with the same electric current. Using high amperages and high amplifications, micro-voltages were successfully converted into clear signals above the background noise level. Since then, LiMCA has been successfully used at moderate temperatures to monitor the quality of molten metals such as gallium, ${ }^{5}$ lead solders, magnesium, ${ }^{6-8}$ zinc, and aluminum..$^{9-11}$ However, the application of LiMCA to the detection of inclusions in melts, such as liquid steel, remains challenging due to the high temperatures that are involved (1500-1700 ${ }^{\circ} \mathrm{C}$ ) and to material problems, such as thermal shock, corrosion, melting, dissolution, and blockage of the small orifice. ${ }^{12}$ In this paper, the LFPA is presented as a method to overcome these challenges.

Lorentz force velocimetry (LFV) is an electromagnetic noncontact flow measurement technique that was invented in the $1960 \mathrm{~s} ;{ }^{13,14}$ it has been recently developed to the point ${ }^{15,16}$ where its application to aluminum production ${ }^{17}$ and steelmaking ${ }^{18}$ is imminent and is being extended as a universal noncontact flow meter for liquids, even liquids with very low electrical conductivity. ${ }^{19}$ LFV is another existing technique that has inspired the LFPA. A permanent magnet in the vicinity of a liquid metal duct or open channel flow enables the flow rate to be derived by measuring the drag force that is exerted on the magnet when the melt flows across the magnetic field. ${ }^{15,16}$ Because it is contactless, this technique can be applied to measure the flow rate of high-temperature melts, such as steels. ${ }^{18}$ However, to date, LFV has never been applied to micron-scale measurement.

\section{BASIC PRINCIPLE}

In this paper, we present an electromagnetic method for detecting and sizing particles that are suspended in the flow of an electrically conducting fluid. Fig. 1 presents a sketch of this method. When a particle-free electrically conducting fluid moves across a magnetic field, as shown in Fig. 1(a), the magnetic field provided by a permanent magnet can be considered a magnetic dipole with moment $\boldsymbol{m}=\mathbf{m} \boldsymbol{e}_{\mathrm{z}}$, whose magnetic field is given by ${ }^{20}$

$$
\boldsymbol{B}(\boldsymbol{R})=\frac{\mu_{0}}{4 \pi}\left\{3 \frac{(\boldsymbol{m} \cdot \boldsymbol{R}) \boldsymbol{R}}{R^{5}}-\frac{\boldsymbol{m}}{R^{3}}\right\},
$$

where $\boldsymbol{R}$ is the space vector from the magnetic dipole, and $R=|\boldsymbol{R}|$. Eddy currents are induced in this electrically 


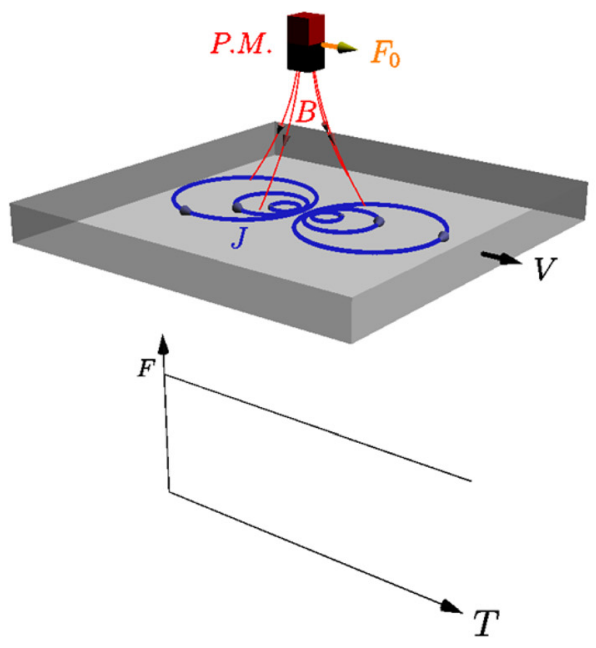

(a)

conducting fluid and can be computed from Ohm's law for a moving electrically conducting fluid

$$
\boldsymbol{J}=\sigma(-\nabla \phi+\boldsymbol{u} \times \boldsymbol{B}),
$$

where $\phi$ is the electrical potential, and $\boldsymbol{u}$ is the fluid velocity. The associated Lorentz force on any unit volume of the moving fluid can be expressed as

$$
\boldsymbol{F}=\boldsymbol{J} \times \boldsymbol{B},
$$

which tends to brake the flow and to reorganize the velocity distribution, as explained in classic textbooks. ${ }^{20-22}$ A reaction force is also exerted on the magnet itself and tends to entrain this magnet in the flow direction. Fig. 1(a) indicates that this global force $\left(F_{0}\right)$ is constant in the case of fluid flow without insulating particles. This force can be measured with a high-resolution force sensor, such as a lasercantilever. $^{23-25}$

Analogous to the ESZ in the $\mathrm{CP}$, we introduce the concept of an electromagnetic sensing zone (EMSZ) to describe the space domain where the magnetic field $\boldsymbol{B}$ and the induced current $\boldsymbol{J}$ are localized within an electrically conducting fluid. Fig. 1(b) shows the case in which an insulating particle is present within the electrically conducting fluid and passes by the magnet. The difference between the electrical conductivities of the two materials produces a spatiotemporal redistribution of the eddy currents $\boldsymbol{J}^{\prime}$ (different from $\boldsymbol{J}$ ) and generates a significant change in the measured force $\left(F_{0}^{\prime}\right)$. The graph of the difference $\Delta F=F_{0}^{\prime}-F_{0}$ versus time exhibits a negative pulse, as shown in Fig. 1(b), and suggests that the presence and size of the particle can be derived from the functional dependence of $\Delta F$ on the particle size. Consequently, a general scaling law can be derived

$$
\Delta F(\boldsymbol{r}, t)=C(\boldsymbol{r}, t) \sigma B^{2} u,
$$

where $C(\boldsymbol{r}, t)$ is a calibrating function depending on the spatial distribution of the magnetic field and the spatiotemporal variation of the electrical conductivity when the particle passes through the EMSZ, $\boldsymbol{r}$ is the space vector, $t$ is the time, $\sigma$ is the electric conductivity of the fluid, $B$ is the magnetic
FIG. 1. Principle sketch of the Lorentz force particle analyzer (LFPA). (a) Action of a small permanent magnet (PM) on the flow of an electrically conducting fluid: a constant force $F_{0}$ aligned with the flow tends to brake the magnetic system; (b) The eddy currents are redistributed when a particle is present in the electromagnetic sensing zone (EMSZ), which produces a negative force pulse. flux density, and $u$ is the relative velocity between the conductor and the magnetic field.

\section{A TWO-DIMENSIONAL NUMERICAL MODEL}

To exhibit physical insight into eddy current redistribution, we have developed a model for an electrically conducting slab that carries a small insulating particle and moves in the presence of a localized magnetic field. For simplification, we use two approximations: (1) instead of a straight slab, we consider a thin rotating metallic ring and (2) we use a frame of reference that moves with this ring. As indicated in Fig. 2, a moving mesh numerical method - Arbitrary LagrangianEulerian $(\mathrm{ALE})^{26}$-is applied to model the relative motion between the magnet and the metallic slab, and the entire domain around this annulus is accordingly divided into two parts: a "stator" that contains the magnet and a "rotor" that rotates with the electrically conducting ring and the insulating particle. The origin of the moving frame is the center $\mathrm{O}$ of the "rotor."

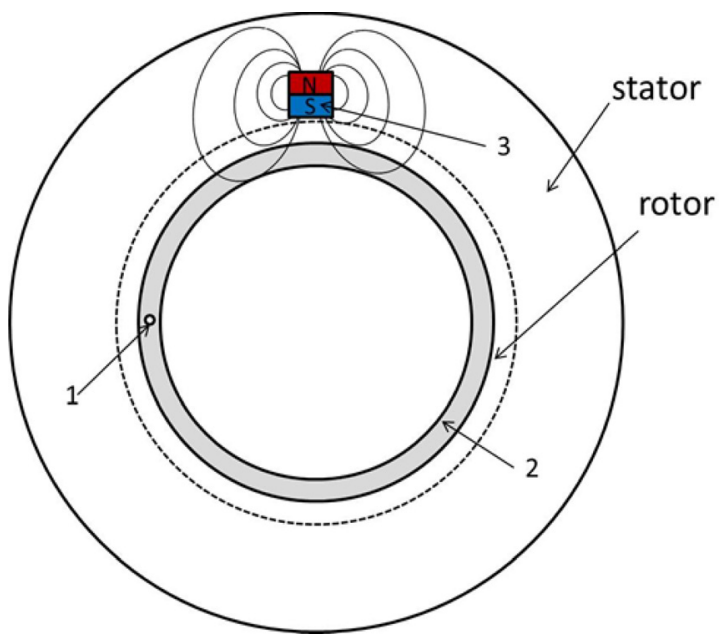

FIG. 2. Configuration of a two-dimensional electromagnetic induction numerical model that demonstrates the features of the LFPA with a deforming mesh method (ALE). 1-particle; 2-copper ring; 3-permanent magnet. The dashed line represents the interface of a moving mesh (rotor) and a static mesh (stator). The rotor rotates in the counterclockwise direction. 
The outer and inner radii of the ring are $25 \mathrm{~mm}$ and $24 \mathrm{~mm}$, respectively. The lengths of the ring's arc and chord are $4.36332 \mathrm{~mm}$ and $4.357787 \mathrm{~mm}$. Their difference $\left(0.0055 \mathrm{~mm}\right.$ or less than $1.3 \times 10^{-3}$ of their average value $)$ is so small that the electromagnetic properties of this circular geometry can be considered almost identical to those of a straight slab because the curvature is very small. As shown in Fig. 2, this ring rotates with an angular velocity of $\omega=4 \pi \mathrm{rad} / \mathrm{s}$ and contains a small insulating particle, whose radius is $200 \mu \mathrm{m}$. The magnet is so small that the EMSZ occupies an angle of only $\pi / 18 \mathrm{rad}$. The distance between the permanent magnet and the surface of the slab is $200 \mu \mathrm{m}$. When the particle passes through this EMSZ, the eddy current distribution and the electromagnetic force that act on the ring change, but the global current remains conserved.

In the steady frame of reference, the $x$-axis is taken as the direction of the magnetization and the $y$-axis as the direction of the ring's motion. The electric potential therefore is oriented in the $z$-direction. As shown in Fig. 2, the coordinates in the moving frame become $X$ and $Y$ and are related to $x$ and $y$ by the following relations:

$$
\left\{\begin{array}{l}
x=\cos (\omega t) \cdot X-\sin (\omega t) \cdot Y \\
y=\sin (\omega t) \cdot X+\cos (\omega t) \cdot Y .
\end{array}\right.
$$

The unsteady 2D magnetic field in the $(X, Y)$ plane can be derived from the time-dependent magnetic vector potential $\boldsymbol{A}=A \boldsymbol{e}_{z}$, such that $\boldsymbol{B}=\nabla \times \boldsymbol{A}$, which has only one nonzero component in the $z$-direction. In the classical approximation for MHD systems, where the magnetic Reynolds number is much smaller than unity, the magnetic field induced by the eddy current can be neglected in comparison with the applied magnetic field. As a consequence, the basic equation for $\boldsymbol{A}$ is

$$
\sigma \frac{\partial \boldsymbol{A}}{\partial \mathrm{t}}+\nabla \times\left(\frac{1}{\mu} \nabla \times \boldsymbol{A}\right)=0 .
$$

In general, such a diffusive vector field would be subject to the skin effect in the conducting domain; however, this effect is negligible in our case. Indeed, the typical transit time of a given part of the ring through the EMSZ is $\tau=L / \omega r \approx 0.013 \mathrm{~s}$; the order of magnitude of the skin depth $\delta$ is significantly larger than the width $(1 \mathrm{~mm})$ of the ring: $\delta=(\tau / \mu \sigma)^{1 / 2} \approx 0.11 \mathrm{~m}$. This means that the ring motion affects the induced current distribution only when the nonconducting particle passes through the EMSZ and concentrates outside this particle to compensate the zero value inside the particle. Consequently, the Lorentz force $\boldsymbol{j} \times \boldsymbol{B}$ is also disturbed. The time-dependent vector potential still obeys Eq. (3) and the classical boundary condition that requires continuity at the stator-rotor interface. The actual magnetic field can then be derived from $\boldsymbol{B}=\mu_{0} \mu_{r} \boldsymbol{H}+\boldsymbol{B}_{r}$ where $\boldsymbol{B}_{r}$ is the remanence of the permanent magnet $\left(B_{r}=1.2 T\right)$.

To solve this problem, we used the COMSOL software package with the ALE method. To guarantee that this simulation is grid-independent, a mesh density was selected that was sufficiently large and uniform, with a minimum size of $1.2 \mu \mathrm{m}$ for any element. The total number of triangular elements was 204126 , the number of degrees of freedom was
470781 , and the time step was set to $2.5 \times 10^{-4} \mathrm{~s}$. The results in the $(X, Y)$ frame were transferred to the $(x, y)$ frame.

Fig. 3 shows how the global Lorentz force, which is integrated over the entire EMSZ, varies with time as the particle moves in front of the magnet. The computed relationship between the force variation and the particle size is shown in the inset of Fig. 3. Note that the negative pulse of the signal is centered exactly at time $t=0.375 \mathrm{~s}$ when the non-conducting particle is located underneath the magnet (also see Fig. 3 inset). The solid lines are iso-values of the current density in the $z$-direction, and the color scale indicates the current in $\mathrm{A} / \mathrm{m}^{2}$. The symmetry is clear when the particle is directly beneath the magnet. The typical width of this signal is approximately three times the width of the permanent magnet, as mentioned above, this is the EMSZ.

\section{PROTOTYPES}

\section{A. Thin flow of GalnSn with water drops}

A small cubic permanent magnet with a length of $1 \mathrm{~mm}$ provided a static magnetic field whose spatial distribution has an essential role. This static magnetic field was computed using the commercial software COMSOL, and the configuration is shown in Fig. 4(a). With a good accuracy, its distribution can be simplified as Gaussian, the probability being $\mathrm{P}[(\mu-\sigma)(\mu+\sigma)]=68.3 \%$, where $\mu$ and $\sigma$ represent the mathematical expectation value and standard deviation, respectively. We chose the standard deviation $\sigma$ as the edge of the EMSZ. By fitting the magnetic field decay curve, we obtained $\sigma_{1}=1.02$ and $\sigma_{2}=0.65$ in the $\mathrm{y}$ - and x-axis directions, respectively. Therefore, the EMSZ has a length of $2.3 \mathrm{~mm}(0.65 \mathrm{~mm} \times 2+1 \mathrm{~mm})$ and a height of $1.02 \mathrm{~mm}$. As depicted in Fig. 4(b), the size of the EMSZ is $2.30 \mathrm{~mm}$ $\times 2.30 \mathrm{~mm} \times 1.02 \mathrm{~mm}$.

The first embodiment of this basic principle is shown in Fig. 5(a). The conducting medium is GaInSn alloy, which is in a liquid state at room temperature. Two automated medical syringes containing GaInSn and water generate a single

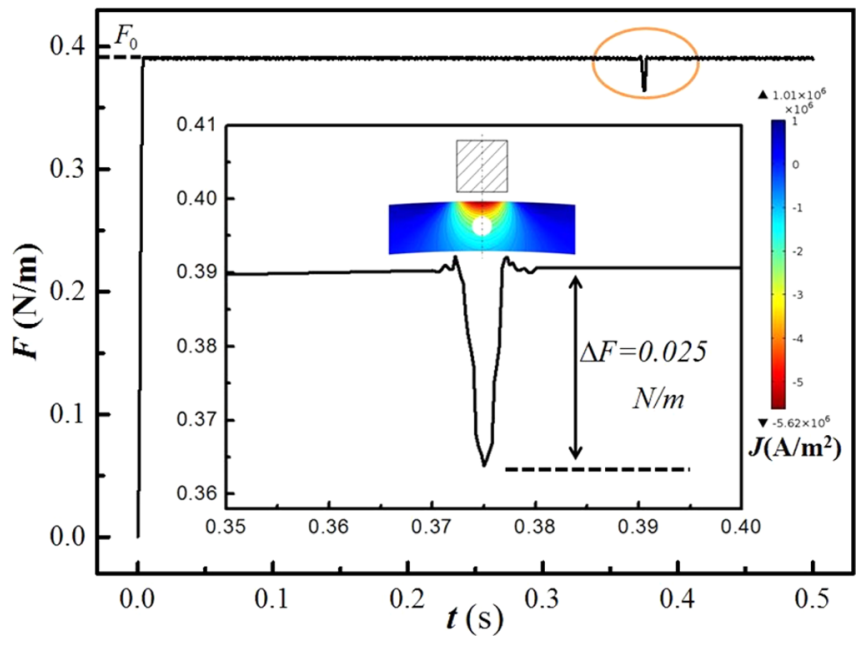

FIG. 3. Graphs of the variation of the Lorentz force $(\Delta F)$ when the insulating particle passes in front of the PM. The inset magnifies the graph, and the contours indicate the equipotential lines of the eddy current when the particle is beneath the PM. 


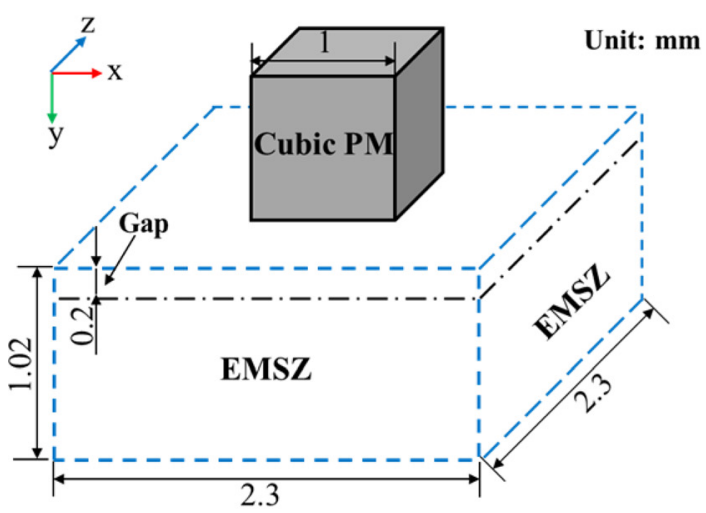

(a)

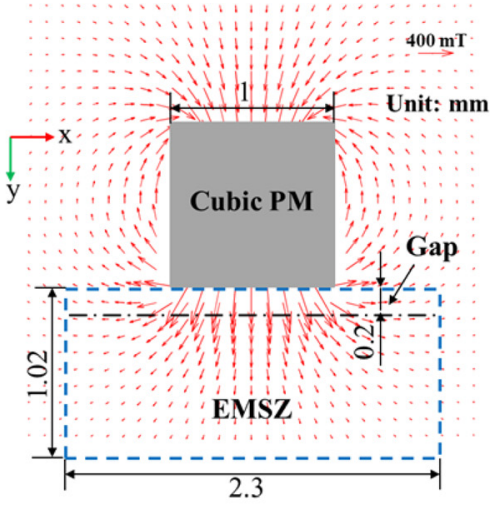

(b)

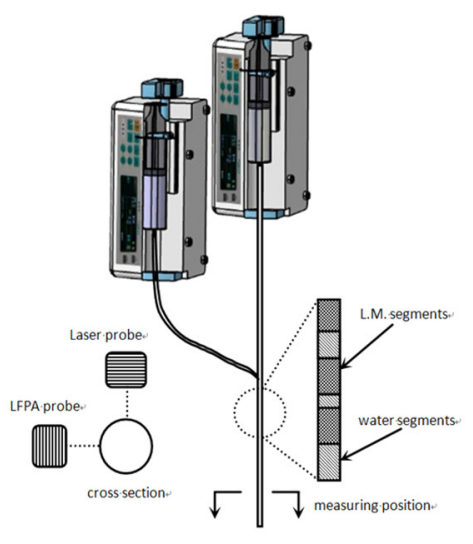

(a)

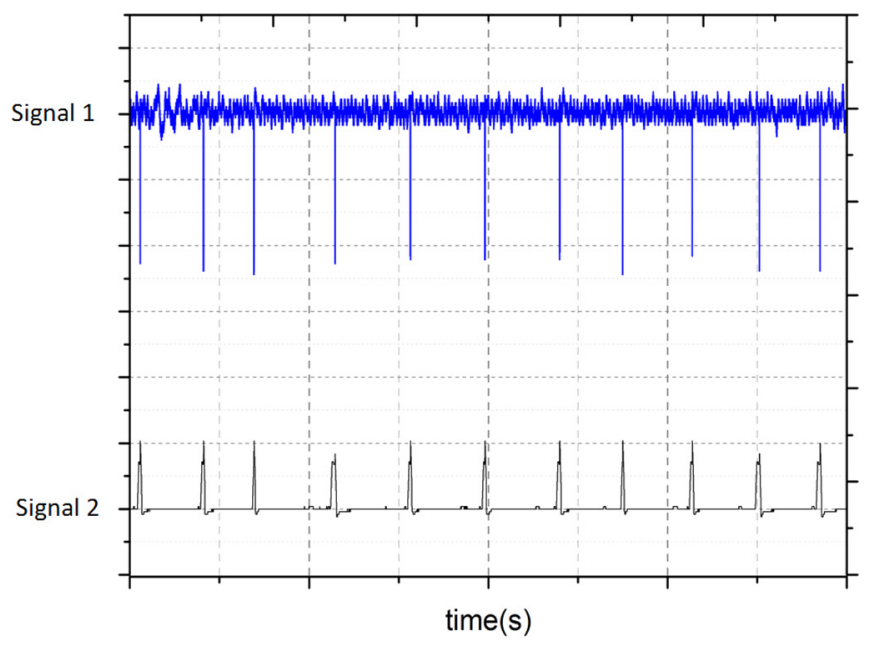

(b)

FIG. 5. (a) Two syringes that contain liquid GaInSn and water are used to generate two-phase particle-laden fluid flow composed of intermittent segments of GaInSn and water. The water mimics insulating particles in an electrically conducting flow. (b) A Lorentz force particle analyzer (LFPA) system was employed to identify and size the water segments (signal 1). An accompanying laser sensor was placed at the same abscissa, perpendicular to the LFPA system, to verify the LFPA results (signal 2). The high correlation of the pulses of the two signals demonstrates the performance of the LFPA in the case in which the diameters of the particles are equivalent to the diameters of the duct. Vertical grid-lines are $400 \mathrm{~ms}$ apart. The average radius of the mimicking particles is $632 \mu \mathrm{m}$. stream of diameter $1.93 \mathrm{~mm}$; the stream is composed of discontinuous GaInSn flows interspersed with short water segments. The electrical conductivities of these two liquids differ by several orders of magnitude, the electrical conductivity of GaInSn being $3.2 \times 10^{6} \mathrm{~S} / \mathrm{m}$ whereas that of water is of the order of $10^{-3} \mathrm{~S} / \mathrm{m}$. These fluid segments mimic well-insulating particles transported by an electrically conducting liquid. The flow rates of GaInSn and water from the syringes were $38.9 \mathrm{~mm}^{3} / \mathrm{s}$ and $3.2 \mathrm{~mm}^{3} / \mathrm{s}$, respectively. The lengths of the two fluid segments were adjusted to simulate the presence of realistic nonconducting particles in a small-diameter liquid metal duct flow.

The reaction of the Lorentz force that acted upon the magnetic-generating-system was measured by a lasercantilever system, which operates with a principle similar to that of an atomic force microscope. ${ }^{23-25}$ The displacement resolution of the laser sensor was $7 \mu \mathrm{m}$. The cantilever was composed of an alloy (12CrNi77) and had a length of $1.5 \mathrm{~mm}$, a width of $1.5 \mathrm{~mm}$, and a thickness of $0.3 \mathrm{~mm}$. The reaction of the Lorentz force yielded a deflection $(\Delta L)$ that was recorded by a laser sensor.

Because GaInSn is opaque and water is transparent, the above system can be checked with another apparatus to obtain comparative information. This apparatus uses a second laser sensor at the same location to record the variations in the lengths of the GaInSn and water segments. Signal 2 in Fig. 5(b) shows the information delivered by the calibrating laser sensor, where low values correspond to water segments and high values correspond to GaInSn segments. Signal 1 in Fig. 5(b) shows the information delivered by the LFPA. The two measurements are remarkably consistent, which demonstrates the feasibility and reliability of the LFPA. As shown in Fig. 5(b), 11 pulse signals appeared within $4 \mathrm{~s}$, and the average radius of the mimicking particles of water drops can be computed $(632 \mu \mathrm{m})$. This experiment also reveals that gas bubble can be sized and counted with the present method.

\section{B. "Dry" prototype compared with a 3d numerical model}

To mimic liquid metal flow, Fig. 6 shows another prototype of the LFPA in which a rotating copper wheel with a diameter of $110 \mathrm{~mm}$, a thickness of $8 \mathrm{~mm}$, and an angular velocity of $17.3 \mathrm{rad} / \mathrm{s}$ was located in the vicinity of a small cubic NdFeB permanent magnet of length $1 \mathrm{~mm}$, whose 


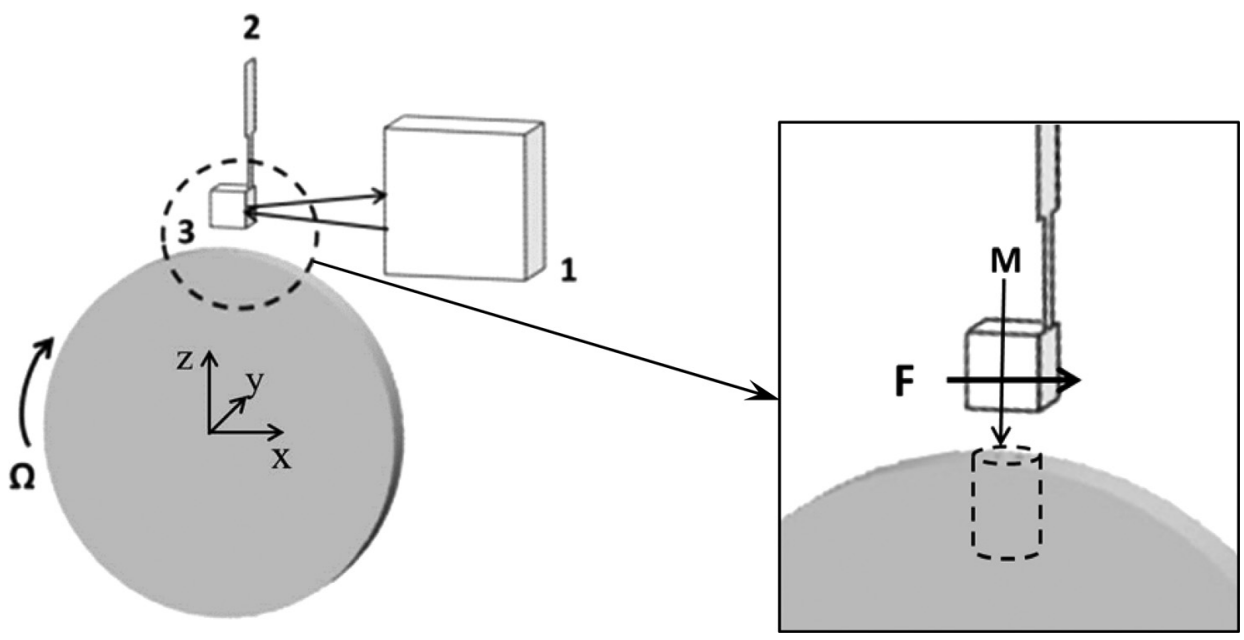

FIG. 6. A rotary copper wheel prototype experiment and its magnified view. A series of holes drilled radially into the edge of a copper wheel to simulate particles in a conducting fluid. The distance between the PM and the edge of the rotating copper wheel is $h=200 \mu \mathrm{m}$. 1-laser sensor, 2-cantilever, 3-PM.

magnetization was perpendicular to the side-edge of the wheel. The other experimental parameters are listed in Table I. A series of cylindrical holes with diameters of 0.5 , $1,2,3$, and $4 \mathrm{~mm}$ and a depth of $0.5 \mathrm{~mm}$ were drilled into the side-edge of the wheel. The magnet and laser-cantilever system were identical to the system shown in Fig. 5.

As shown in Fig. 7, the deflection $(\Delta F)$ due to the Lorentz force acting on the magnet was recorded by the laser sensor; the deflection appears to be dependent on the hole volume V. Again, this prototype experiment demonstrates that the LFPA is suitable for detecting and sizing insulating particles or defects in a moving electrical conductor.

Additionally, to verify the results derived with this prototype, we developed a three-dimensional numerical model using the software package COMSOL Multiphysics in the situation shown in Fig. 6. In this simulation, the electrical conductor is drilled with a cylinder that mimics the insulating particle and moves with a constant velocity $\mathrm{v}$ in the vicinity of the magnet; the eddy currents are calculated with Ohm's law (Eq. (2)). The origin of the frame is shown in Fig. 6. The results that represent the motion of the non-conducting cylinder through the EMSZ are easily obtained.

In this three-dimensional numerical model, the magnetization direction of the permanent magnet, the direction of motion of the plate, and its velocity were the same as in the experiment shown in Fig. 7. A vibration numerical model was employed to determine the relationship between the force acting upon the magnetic-generating-system and the deflection of the laser-cantilever used in the present experiments. For conciseness, we do not discuss details on this technique here; they will be presented in a future paper. ${ }^{27}$

TABLE I. Parameters in the prototype (Fig. 6).

\begin{tabular}{lc}
\hline \hline Parameter & Values \\
\hline Dimensions of the permanent magnet $(\mathrm{mm})$ & $1 \times 1 \times 1$ \\
Velocity of the copper wheel $(\mathrm{m} / \mathrm{s})$ & 0.95 \\
Electrical conductivity of the copper wheel $(\mathrm{S} / \mathrm{m})$ & $5.792 \times 10^{7}$ \\
Dimension of the cylindrical mimicking particle $(\mathrm{mm})$ & $\Phi D \times 0.5$ \\
Dimension of the copper wheel $(\mathrm{mm})$ & $\Phi 50 \times 5$ \\
Remanence of the permanent magnet $(T)$ & 1.2 \\
Distance between the permanent magnet and plate $(\mathrm{mm})$ & 0.2 \\
\hline \hline
\end{tabular}

Fig. 7 shows the eddy current redistribution evolution for a cross-section through $z=24.9 \mathrm{~mm}$ (see Fig. 6) when a particle passes through the EMSZ due to the presence of a permanent magnet. The arrows indicate the eddy current vectors. In the absence of the particle, the eddy currents should form a pair of vortices with the opposite directions. We denote the time of the moving wheel at the point just beneath the vertical center of the PM (see Fig. 6) by $T_{0}$. The results of the eddy current redistribution influenced by the particle are illustrated in Figs. 7(a)-7(e) at five typical times. The arrows indicate the eddy current vectors, and the color scale indicates the current in $\mathrm{A} / \mathrm{m}^{3}$. The times shown in Figs. 7(a)-7(e) are $t=\left(T_{0}\right.$ $-1.5) \mathrm{ms}, \quad t=\left(T_{0}-0.5\right) \mathrm{ms}, \quad t=T_{0} \mathrm{~ms}, \quad t=\left(T_{0}+0.5\right) \mathrm{ms}$, and $t=\left(T_{0}+1.5\right) \mathrm{ms}$, respectively. Small asymmetries in the current distribution when the particle enters and exits the EMSZ are shown in Figs. 7(a) and 7(e), respectively. Conversely, the symmetry is clear when the particle is directly beneath the magnet, as shown in Fig. 7(c). Figs. 7(b) and 7(d) show both a significant asymmetry and an increase in $j_{z}$ in the immediate vicinity of the particle, which is necessary to compensate its zero value within the particle. The entire process is similar to solar and lunar eclipses. Additionally, the images span $3 \mathrm{~ms}$; with a velocity of $0.95 \mathrm{~m} / \mathrm{s}$, the equivalent distance is $2.85 \mathrm{~mm}$, which is approximately 3 times the length of the PM, validating the typical length of the EMSZ shown in Fig. 4. The maximum eddy current density reaches $3.53 \times 10^{6} \mathrm{~A} / \mathrm{m}^{3}$.

Comparisons of the experimental and numerical results for the variation of $\Delta F$ versus the particle volume $\mathrm{V}$ are shown in Fig. 8(a) and Table II. The amplitude of the negative pulse $\Delta F$ is an important ingredient in the measuring process of the LFPA despite its small size: the amplitude of $\Delta F$ is $10^{-7}-10^{-4} N$ for micron-sized particles. The force variation $\Delta F$ decreases substantially with particle volume.

In Eq. (4), the constant $C(\boldsymbol{r}, t)$ can be written

$$
C(\boldsymbol{r}, t) \sim \pi r^{2} h \hat{C}(t)
$$

where $h$ is the depth of the cylinder, $\pi r^{2} h$ is the volume of the small hole, and $\hat{C}(t)$ denotes a type of pulse function, analogous to the probability density of a normal distribution. To verify this relationship, we have conducted appropriate experiments allowing a comparison with the results in Table II, also shown in Fig. 8(a). The corresponding experimental 


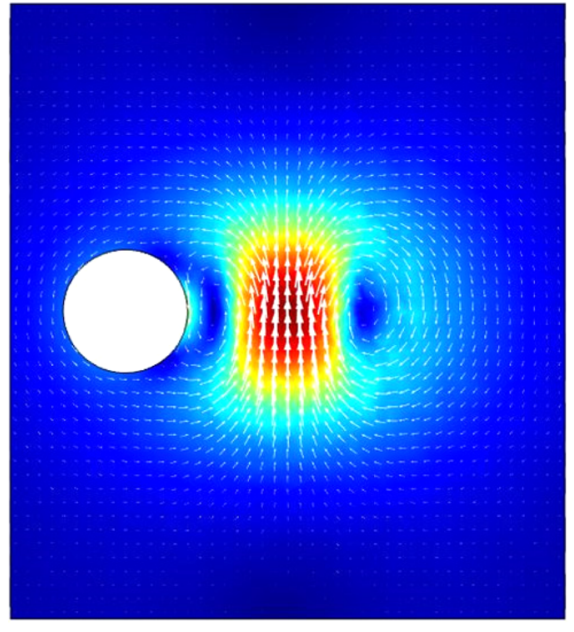

(a)

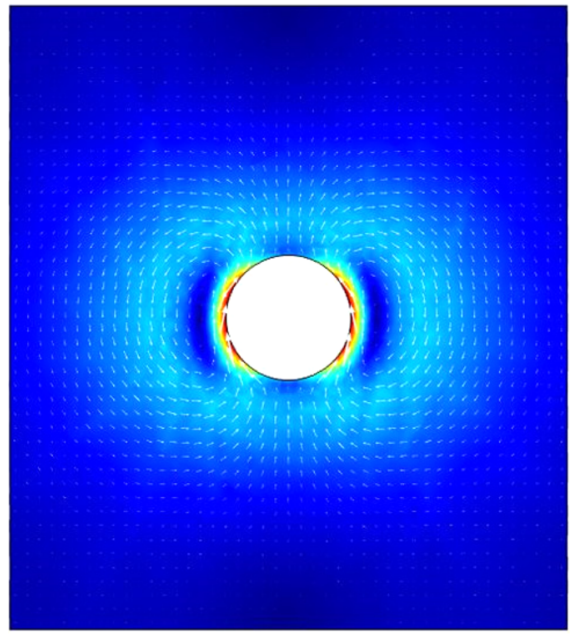

(c)

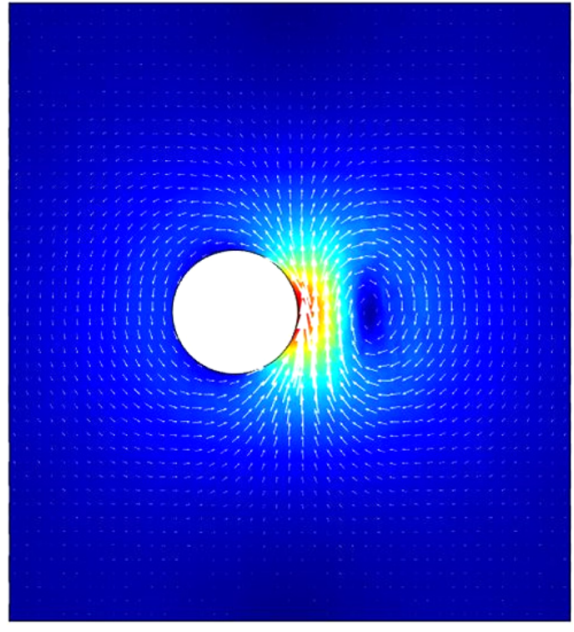

(b)

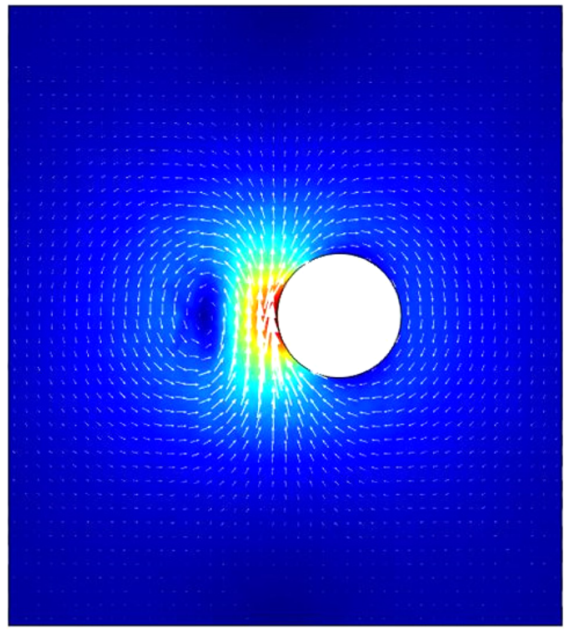

(d)

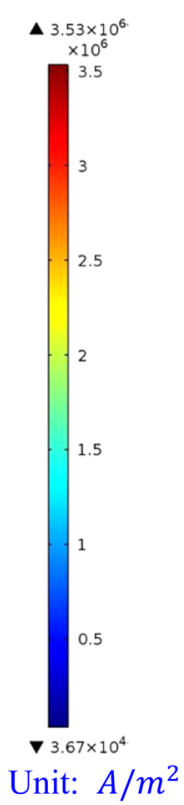

7. Eddy current redistribution evolution for a cross-section at $z=24.9 \mathrm{~mm}$ (see Fig. 6) when a particle passes through the EMSZ due to a permanent magnet. The arrows indicate the eddy current. We denote the time of the moving wheel at the point just beneath the vertical center of the PM (see Fig. 6) by $T_{0}$. The five images span $3 \mathrm{~ms}$ : (a) $\left(\mathrm{T}_{0}-1.5\right) \mathrm{ms}$, (b) $\left(\mathrm{T}_{0}-0.5\right) \mathrm{ms}$, (c) $\mathrm{T}_{0} \mathrm{~ms}$, (d) $\quad\left(\mathrm{T}_{0}+0.5\right) \mathrm{ms}$, and (e) $\left(\mathrm{T}_{0}+1.5\right) \mathrm{ms}$.

(e)

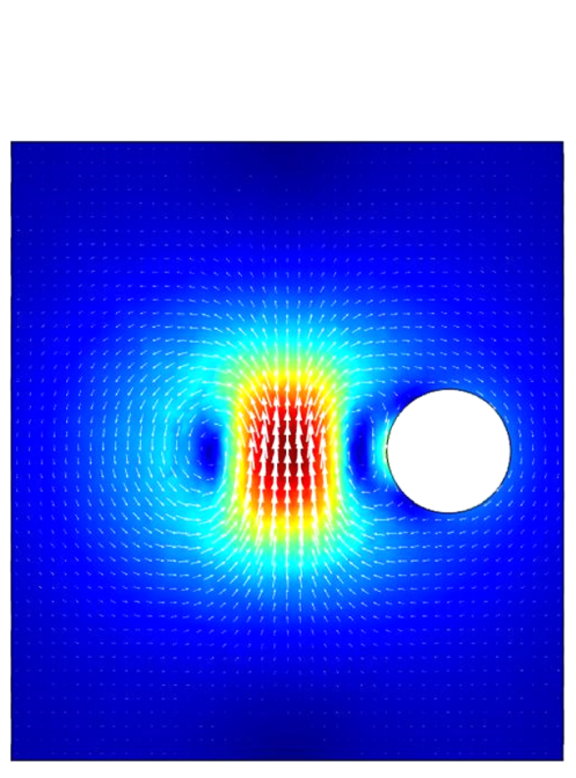

parameters are identical to the parameters of the numerical simulation. As shown in Fig. 8(a), the experimental data and the three-dimensional numerical results are in fair agreement and confirm the feasibility of LFPA.
Therefore, this three-dimensional numerical model demonstrates the ability to simulate this problem. Though the drilling of such small holes to mimic the insulating particles was difficult, this experiment demonstrates the possibility to 


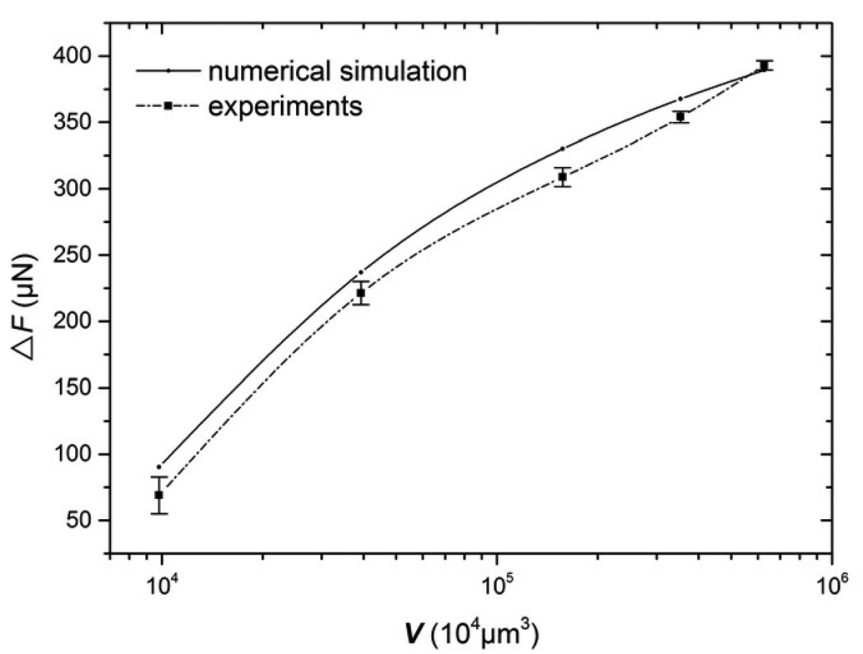

(a)

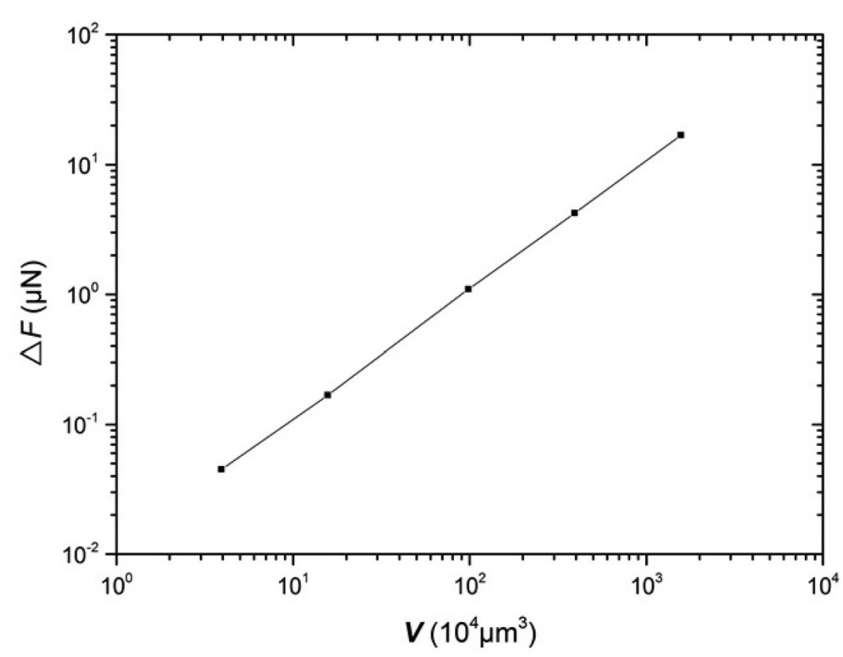

(b)

FIG. 8. Experimental and numerical results of the relationship between the variation in the reaction force acting upon the PM and particle size over (a) a large range $(d=0.5,1,2,3$ and $4 \mathrm{~mm})$ and (b) a small range $(d=10$, $20,50,100$ and $200 \mu \mathrm{m})$ of particle size.

simulate this problem physically. Indeed, we have been able to investigate the functional variation of $\Delta F$ versus $\mathrm{V}$ in the range from $10 \mu \mathrm{m}$ to $200 \mu \mathrm{m}$ using this three-dimensional numerical model; the results are shown in Fig. 8(b). This finding reveals that with the distribution of the magnetic field as previously discussed in Section IV, and in the range of

TABLE II. Calculated values of $\Delta F$ for various particles.

\begin{tabular}{lcc}
\hline \hline Diameter $(\mu \mathrm{m})$ & Volume $\left(\times 10^{4} \mu \mathrm{m}^{3}\right)$ & $\Delta F(\mu \mathrm{N})$ \\
\hline 10 & 3.926991 & 0.190 \\
20 & 15.70797 & 0.950 \\
50 & 98.17475 & 1.933 \\
100 & 392.6991 & 5.070 \\
200 & 1570.797 & 17.712 \\
500 & 9817.475 & 91.200 \\
1000 & 39269.91 & 237.828 \\
2000 & 157080.0 & 331.032 \\
3000 & 353430.0 & 368.413 \\
4000 & 628320.0 & 390.189 \\
\hline \hline
\end{tabular}

particle size from $10 \mu \mathrm{m}$ to $200 \mu \mathrm{m}$, the relationship is approximately linear. Note the extremely low forces to be measured, of the order of $10^{-7} N$ for a particle of $10 \mu \mathrm{m}$. This requires a difficult and sensitive measurement, requiring special techniques such as the AFM. ${ }^{23-25}$

\section{DISCUSSION AND CONCLUSION}

We have presented the principle of LFPA, a contactless electromagnetic diagnostic method, two prototype experiments, and two numerical simulations to verify the feasibility of the method at various scales. As demonstrated above, LFPA possesses the ability to measure a small force. The experiment depicted in Figure 4 demonstrates the detection of $200 \mu \mathrm{m}$ particles in a metal whose electrical conductivity and force to be measured are on the order of $10^{7} \mathrm{~S} / \mathrm{m}$ and $10^{-4} \mathrm{~N}$, respectively. This challenge appears straightforward when using a laser-cantilever system. ${ }^{23-25}$ Because this force is proportional to the melt electrical conductivity, ${ }^{15,16,22}$ the technique should also be effective in semiconducting materials whose conductivity is of the order of $10^{6} \mathrm{~S} / \mathrm{m}$. The force in that case, however, is of the order of $10^{-8} \mathrm{~N}$ in electrolytes where conductivity is approximately $10^{5}$ times smaller than the conductivity of metals; this hinders measurement.

The LFPA presented in this paper is a new application of this type of electromagnetic device. It might suggest successful improvements of existing technologies that require significant purity of liquid or solid metallic alloys. For example, in aluminum and steel continuous casting devices, any inclusion of oxide should be detected and sized online, without any contact, at the entry into the mold.

\section{ACKNOWLEDGMENTS}

The authors are grateful to the "One Hundred Talented People" program of The Chinese Academy of Sciences, the National Natural Science Foundation of China (51374190), and the Chinese Academy of Science (CAS) Project (YZ201567) for financial support of this study.

\footnotetext{
${ }^{1}$ A. Moldavan, Science 80, 188-189 (1934).

${ }^{2}$ W. H. Coulter, U.S. patent 2656508 (1953).

${ }^{3}$ M. D. Graham, JALA 8(6), 72 (2003).

${ }^{4}$ D. Doutre and R. Guthrie, U.S. patent 4555662 (1985).

${ }^{5}$ D. Doutre, Ph.D. thesis, McGill University, Montreal, 1984.

${ }^{6}$ H. Nakajima and R. I. L. Guthrie, U.S. patent 5,198,749 (1993).

${ }^{7}$ C. Carozza, P. Lenard, R. Sankaranayanan, and R. I. L. Guthrie, Proceedings of the 36th Annual Conference of Metallurgists of CIM-Light Metals Symposium, Sudbury, Ontario, Canada, August 1997 (Canadian Institute of Mining, Metallurgy and Petroleum), pp. 185-196.

${ }^{8}$ R. I. L. Guthrie, U.S. patent 5789910 (1998).

${ }^{9}$ M. Li and R. I. L. Guthrie, Metall. Mater. Trans. B 31, 357 (2000).

${ }^{10}$ R. I. L. Guthrie and M. Li, Metall. Mater. Trans. B 32, 1067 (2001).

${ }^{11}$ R. I. L. Guthrie and M. Li, Metall. Mater. Trans. B 32, 1081 (2001).

${ }^{12}$ X. D. Wang, M. Isac, and R. I. L. Guthrie, ISIJ 49(7), 975-984 (2009).

${ }^{13}$ J. A. Shercliff, The Theory of Electromagnetic Flow Measurement (Cambridge University Press, Cambridge, 1962).

${ }^{14}$ J. A. Shercliff, U.K. patent GB 831226 (1960).

${ }^{15}$ A. Thess, E. Votyakov, and Y. Kolesnikov, Phys. Rev. Lett. 96, 164501 (2006).

${ }^{16}$ A. Thess, E. Votyakov, B. Knaepen, and O. Zikanov, New J. Phys. 9, 299 (2007).
} 
${ }^{17}$ Y. Kolesnikov, C. Karcher, and A. Thess, Metall. Mater. Trans. B 42, 441-450 (2011).

${ }^{18}$ D. Jian, C. Karcher, X. Xu, A. Deng, E. Wang, and A. Thess, in 7th International Congress on Electromagnetic Processing of Materials, Beijing, China (2012).

${ }^{19}$ A. Wegfrass, Ch. Diethold, M. Werner, Th. Froehlich, B. Halbedel, F. Hibrunner, Ch. Resagk, and A. Thess, Appl. Phys. Lett. 100, 194103 (2012).

${ }^{20}$ R. Moreau, Magnetohydrodynamics (Kluwer Academic Publishers, Dordrecht, 1990).

${ }^{21} \mathrm{P}$. H. Roberts, An Introduction to Magnetohydrodynamics (Elsevier, New York, 1967).
${ }^{22} \mathrm{P}$. A. Davidson, An Introduction to Magnetohydrodynamics (Cambridge University Press, Cambridge, 2001).

${ }^{23}$ G. Binnig, H. Rohrer, C. Gerber, and E. Weibel, Phys. Rev. Lett. 49(1), 57-61 (1982).

${ }^{24}$ G. Binnig, H. Rohrer, Ch. Gerber, and E. Weibel, Phys. Rev. Lett. 50, 120-123 (1983).

${ }^{25}$ G. Binnig, H. Rohrer, Ch. Gerber, and E. Weibel, Appl. Phys. Lett. 40(2), 178-180 (1982)

${ }^{26}$ T. J. R. Hughes, W. K. Liu, and T. K. Zimmerman, Comput. Methods Appl. Mech. Eng. 29, 329-340 (1981).

${ }^{27}$ Unpublished results of numerical calibration of Lorentz force particle analyzer using laser-cantilever system. 